\title{
Research of Nonlinear Vibration of Aero-Engine in Windmilling Imbalance with Wing Degree-of-freedom
}

\author{
Shuang LIU $^{1, \text { a }}$, Wei WANG ${ }^{2, b}$ \\ ${ }^{1}$ Civil Aircraft Airworthiness Certification Technology and Management Research Center, Civil \\ Aviation University of China, Tianjin, 300300, China \\ ${ }^{2}$ Civil Aircraft Airworthiness Certification Technology and Management Research Center, Civil \\ Aviation University of China, Tianjin, 300300, China
}

aemail: 51458841@qq.com, bemail:weiwangcauc@126.com

Keywords: windmilling; nonlinear vibration; wing degree-of-freedom; rotor/stator system

\begin{abstract}
The rotor non-isotropic produced nonlinear vibration of aero-engine in windmilling would cause serious danger to flight security. So the research of vibration character in windmilling should go further. In this paper, the kinematic equation of the rotor/stator/wing system is established based on Jeffcott rotor and flexible supported stator interaction system. Afterwards, the nonlinear kinematic differential equation is solved with state space method and both the amplitude of wing and orbit of rotor/stator in different twirling speed are obtained. The result has presented that the rotor non-isotropic will evoke larger amplitude of rotor/stator in lower windmilling speed and increase the probability of flight disaster if wing DOF is considered.
\end{abstract}

\section{Introduction}

Aero-engine windmilling refers a metastable status that the aerodynamic force, rotor inertia and resistance torque drive the engine rotor shaft to rotate and maintain at one rotating speed during a short time when the engine stops in flight [1]. In the condition of windmilling, the low frequency and high amplitude vibration are induced by the unbalanced force when a fan-blade comes off can lead to a series of negative consequences to the rotor system and even endangered flight safety[2].The engine must be kept within windmilling in diversion time after a fan-blade failure. Experiment on this condition isn't feasible by reason of the complex work environment of aero-engine and flight safety. So, in order to get the system features of this flight condition closer to the reality, more accurately numerical analysis must be carried out.

At present, many scholars have detailedly studied in vibration of aero-engine rotor system in windmilling condition. Their studies are principally distributed in rotor vibration characteristics, dynamic performance of the bearing, reliability, and so on, but not covered the actual situation that the engine is hanged on the wing. And there is no specific research results show that whether new vibration behavior of the rotor system will appear and what kind of amplitude change happens after considering the wing DOF. Because of the engine vibration characteristics closely associating with flight safety, it is very important to study the influence of wing DOF on the vibration characteristics of the rotor system.

In this paper, windmilling imbalance nonlinear vibration model including wing DOF is established based on the model provided by Gotz von Groll [3] and the maximum vibration amplitudes of the wing when a wing DOF is considered and not are compared through the simulation results. The possibility of disastrous consequences caused by the resonance of the wing/engine system aroused by the reason of geometric/stiffness asymmetry in windmilling is verified. The relevant qualitative conclusions may provide the engine and aircraft manufacturers a reference for the design of engine structure and shock-absorbing.

\section{Vibration model of aero-engine in windmilling imbalance}

Rotor/stator system model. The main objective of this paper is to analyze the change by the 
vibratory characteristic of the rotor/stator system by the wing DOF. Neglecting the other factors, the model of rotor/stator interaction system would be simulated by Jeffcott rotor and stator supported by spring which presented by Gotz von Groll. Only applying a single disc rotor, the model of the rotor/stator system is established as a circular and rigid body without the elastic deformation. In the process of numerical simulation, a small contact penetrative area is included between the rotor and stator. Generally, the value of the contact stiffness is orders of magnitudes higher than the rotor or stator support stiffness in the contact penetrative area, then the order of magnitudes of the contact depth is much lower than one of the displacement of the rotor or stator [3].So the contact penetrative area of the rotor and stator can be simplified as a single point in mathematics [4]. The contact depth is defined as:

$$
\delta(t)=\left\{\begin{array}{l}
r_{r}+\varepsilon_{r} e^{i \omega t}-r_{s}-\varepsilon_{s}-h e^{i \psi} \quad\left|r_{r}+\varepsilon_{r} e^{i \Omega t}-r_{s}-\varepsilon_{s}\right|>h \\
0
\end{array}\right.
$$

Where the displacements of the rotor and stator in the complex plane are $r_{r}$ and $r_{s}$, respectively. $h$ is the gap size, $\psi$ is the angular position of contact, $\varepsilon_{r}$ and $\varepsilon_{s}$ are the geometric offset of rotor and stator, respectively. They are shown in Fig.1.

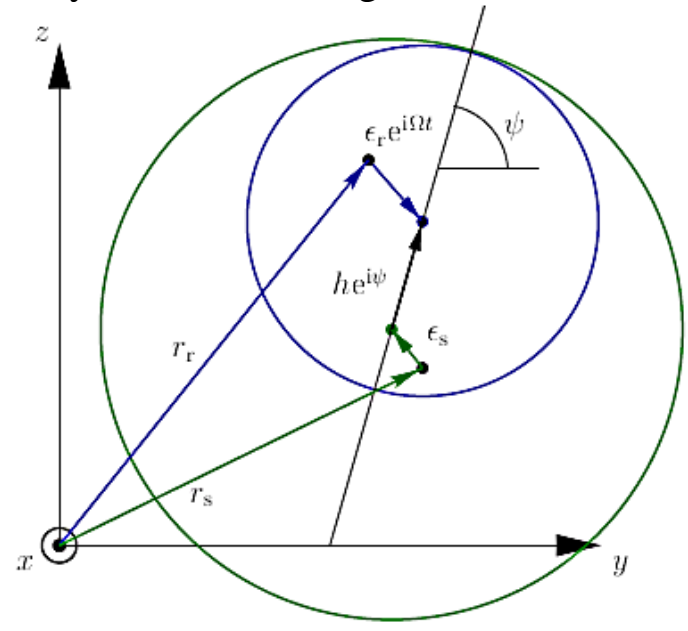

Fig.1. Geometry of rotor/stator model

Contact force model. In practice, the contact force is very complicated in a contact system, it depends on the geometric construction, the properties of materials, the impact depth, the speed of the objects and etc. [5]. The common contact force are shown below.

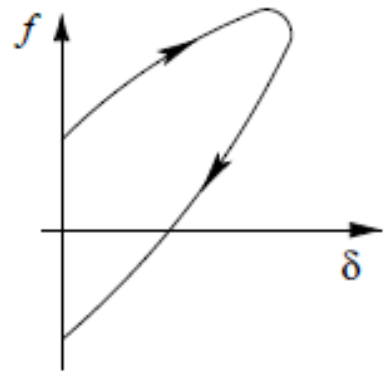

(a) linear

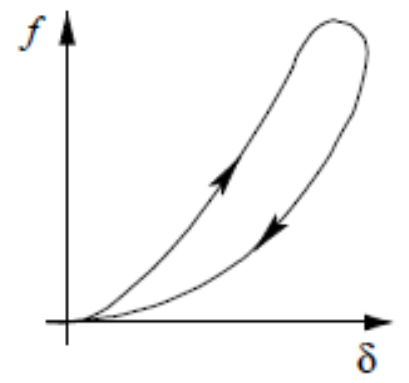

(b) nonlinear

Fig.2. Force-displacement characteristics

Suppose $f_{n}$ to be the normal contact force between the rotator and stator, and the relationship can be simplified as a linear expression numerically:

$$
f_{n}=k_{c} \delta+c_{c} \dot{\delta}
$$


Where $\delta$ is the contact depth in Eq.(1), $\dot{\delta}$ is the derivative of $\delta$ with respect to time, $k_{c}$ is the partial linear contact stiffness, $c_{c}$ is the partial damping coefficient. From Fig.2.(a), it figures out two problems about the linear model: (1) when the contact displacement comes back to 0 , the negative contact force in the model is impractical; (2) the curve cannot be differentiated at $\delta=0$. The common numerical calculation program cannot deal with the in-differentiable or discontinuous function. The numerical calculation is hard to converge when applying this model, thus, the damping coefficient of the model is subjected to $c_{c}=0$.

Further, in practice, the fraction is probably be either simple or complicated, the paper chooses the simple Coulomb fraction model [6]. In which, $\mu$ is denoted to a constant fraction coefficient and the total contact force, $f_{c}$, is shown in complex form as:

$$
f_{c}=(1+i \mu) f_{n}
$$

The viscous fraction is neglected in windmilling condition, since the gap size, h, is much smaller than the outer diameter of the rotor [7].

Motion equations of rotor/stator system. Accounting for the discussion above, the equations of emotion of the multi-degree-of-freedom rotator/stator system is denoted to the complex form:

$$
\begin{aligned}
& m_{r} \ddot{r}_{r}+c_{r} \dot{r}+k_{r} r_{r}=-f_{c}+\Omega^{2} m_{r} \varepsilon_{m} e^{i \Omega t} \\
& m_{s} \ddot{r}_{s}+c_{s} \dot{r}_{s}+k_{s} r_{s}=f_{c}
\end{aligned}
$$

Where $m_{r}$ is the rotor mass, $m_{s}$ is the stator mass, $\Omega$ is the speed of the axis. The parameters below is included:

$$
\omega_{r}^{2}=\frac{k_{r}}{m_{r}}, \omega_{s}^{2}=\frac{k_{s}}{m_{r}} \equiv \gamma_{k} \omega_{r}^{2} \quad \zeta_{r}=\frac{\omega_{r} c_{r}}{2 k_{r}}, \quad \zeta_{s}=\frac{\omega_{s} c_{s}}{2 k_{s}}, \quad \gamma_{m}=\frac{m_{s}}{m_{r}} \quad \gamma_{k}=\frac{k_{s}}{k_{r}} \quad \gamma_{c}=\frac{k_{c}}{k_{r}}
$$

substitute into Eq.(4) and Eq.(5):

$$
\begin{aligned}
& \ddot{r_{r}}+2 \zeta_{r} \omega_{r} \dot{r}_{r}+\omega_{r}^{2} r_{r}=-\omega_{r}^{2}(1+i \mu) \gamma_{c} \delta+\Omega^{2} \varepsilon_{m} e^{i \Omega t} \\
& \ddot{\gamma}_{m} \ddot{r}_{s}+2 \zeta_{s} \omega_{s} \dot{r}_{s}+\omega_{s}{ }^{2} r_{s}=\omega_{r}^{2}(1+i \mu) \gamma_{c} \delta
\end{aligned}
$$

Note that the coefficients above are dealt by the normalization approach with respect to the rotor parameters [8]. For example, the stator parameter, $\omega_{s}$, which has been processed with respect to $m_{r}$, is capable to be directly compared with the result of some models without the stator mass $m_{s}$, in the reference.

\section{Vibration model in windmilling imbalance involving wing degree-of-freedom}

It is shown that compared with actual flight status, the four DOFs dynamic model established based on rigid rotor and flexible supported stator was greatly simplified through the introduction. Some differences may exist between the vibration characteristics of rotor and stator obtained by dynamic model simulation and the actual situation. Thus the wing DOF were introduced extra and then a more practical dynamic model can be got.

Because the vibration amplitude of the wing in vertical direction is far greater than the horizontal and expand direction, the wing DOF in the vertical direction was only considered in this paper. 
Accordingly, some necessary modifications should be made to the dynamic model of the wing and engine system on the basis of the rotor and stator system. These modifications can also be made through Fig.1, in which the displacement of the ground position of the rotor and stator support was replaced by the vertical vibration motion of the wing. Reflected in the equations, the influences of vertical direction vibration of the wing $-i \dot{r}_{\text {wing }}$ were added to Eq,(4) and Eq.(5). Then the motion equations of the wing and engine system can be obtained as follow:

$$
\begin{aligned}
& m_{r}\left(\ddot{r}_{r}-i \ddot{r}_{\text {wing }}\right)+c_{r}\left(\dot{r}_{r}-i \dot{r}_{\text {wing }}\right)+k_{r}\left(r_{r}-i r_{\text {wing }}\right)=-f_{c}+\Omega^{2} m_{r} \varepsilon_{m} e^{i \Omega t} \\
& m_{s}\left(\ddot{r}_{s}-i \ddot{r}_{\text {wing }}\right)+c_{s}\left(\dot{r}_{s}-i \dot{r}_{\text {wing }}\right)+k_{s}\left(r_{s}-i r_{\text {wing }}\right)=f_{c} \\
& m_{\text {wing }} \ddot{r}_{\text {wing }}+k_{\text {wing }} r_{\text {wing }}=\Omega^{2} m_{r} \varepsilon_{m} e^{i \Omega t}
\end{aligned}
$$

After some processes similar to the second part, the equations can be written as:

$$
\begin{aligned}
& \left(\ddot{r}_{r}-i \ddot{r}_{\text {wing }}\right)+2 \zeta_{r} \omega_{r}\left(\dot{r}_{r}-i \dot{r}_{\text {wing }}\right)+\omega_{r}^{2}\left(r_{r}-i r_{\text {wing }}\right)=-\omega_{r}^{2}(1+i \mu) \gamma_{c} \delta+\Omega^{2} \varepsilon_{m} e^{i \Omega t} \\
& \gamma_{m}\left(\ddot{r}_{s}-i \ddot{r}_{\text {wing }}\right)+2 \zeta_{s} \omega_{s}\left(\dot{r}_{s}-i \dot{r}_{\text {wing }}\right)+\omega_{s}^{2}\left(r_{s}-i r_{\text {wing }}\right)=\omega_{r}^{2}(1+i \mu) \gamma_{c} \delta \\
& \gamma_{\text {wing }} \ddot{r}_{\text {wing }}+\omega_{\text {wing }}^{2} r_{\text {wing }}=\Omega^{2} m_{r} \varepsilon_{m} e^{i \Omega t}
\end{aligned}
$$

in Eq.(13),

$$
\gamma_{\text {wing }}=\frac{m_{\text {wing }}}{m_{r}}, \omega_{\text {wing }}^{2}=\frac{k_{\text {wing }}}{k_{r}} \omega_{r}^{2}
$$

The commonly used state-space numerical algorithm is chosen to solve the differential equation upstairs. Formulating a problem in state space one obtains:

$$
\{\dot{q}\}=[A]\{q\}+[B]\{f(t, q)\}
$$

in which

$$
\{q\}=\left(\begin{array}{l}
r \\
\dot{r}
\end{array}\right) \quad[A]=\left[\begin{array}{lc}
0 & I \\
-[M]^{-1}[K] & -[M]^{-1}[C]
\end{array}\right] \quad[B]=\left[\begin{array}{c}
0 \\
{[M]^{-1}}
\end{array}\right]
$$

As state space equation has been listed, Runge-Kutta single step method is applied to measuring time integration and conservation, which are all coded in MATLAB.

\section{Simulation study of wing DOF effecting on vibration of aero-engine in windmilling}

In order to make the simulation result play well as a reference, this paper uses the same parameter with the research of Gotz von Groll's so that the simulation result can be compared to the reference documents. Meanwhile, this paper adds two parameters to express the wing DOF, as in Table 1.

After simulation with the given parameters, the trajectory of wing, rotor and stator, variance of vibration amplitude with rotation speed and frequency response can be got. Fig.3 shows the comparison of the maximum vibration amplitude of the wing when a wing DOF is considered and not. Fig. 4 and Fig.5 show the trajectory of rotor and stator with wing DOF and not, and Fig. 6 and Fig.7 bring out the result of the spectral response when wing DOF is considered and ignored. 
Table.1. parameters used in simulation

$\begin{array}{cccc}\frac{m_{\text {wing }}}{m_{r}}=3 & \frac{k_{\text {wing }}}{k_{r}}=1.6 & \varepsilon_{m}=1 e^{i \frac{\pi}{2}} & \Omega=0.001 \text { rad / } \\ \omega_{r}=1 \mathrm{rad} / \mathrm{s} & \omega_{s}^{2}=\gamma_{k} \omega_{r}^{2} & \zeta_{r}=0.05 & \zeta_{S}=0.05 \\ \gamma_{m}=0.5 & \gamma_{k}=2 & \gamma_{c}=20 & c_{c}=0 \\ \mu=0.1 & h=2 & \varepsilon_{r}=0 & \varepsilon_{s}=e^{i \frac{\pi}{2}}\end{array}$

By the comparison to the simulation result when a wing DOF is considered (as in Fig.3), although the rotation speed of stator is low when engine is in windmilling, there is still possibility that the asymmetry of geometric or stiffness leads to resonance of the wing/engine system at low frequencies $\left(\Omega / \omega_{r} \approx 0.75\right)$. It can be confirmed by the trajectory of rotor and stator in Fig.4 ( $\Omega / \omega_{r} \approx 0.7$ and $\Omega / \omega_{r} \approx 0.75$ ). Meanwhile, Fig.3 shows that the resonance response frequency $\Omega / \omega_{r} \approx 0.75$ at this time is less than the natural frequency of the wing $\omega_{\text {wing }} / \omega_{r}=\sqrt{\frac{m_{\text {wing }} k_{r}}{k_{\text {wing }} m_{r}}}=1.370$.

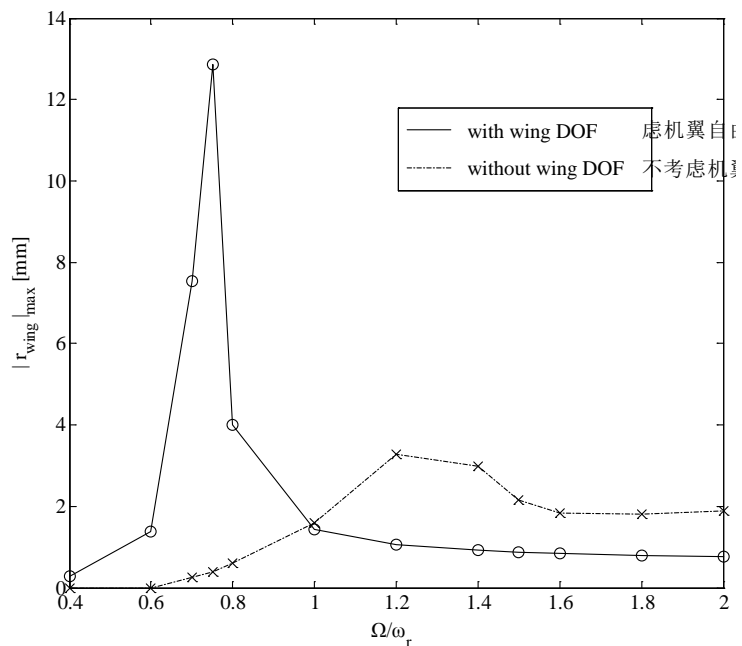

Fig.3. The maximum amplitude of wing comparison when wing DOF is considered and ignored
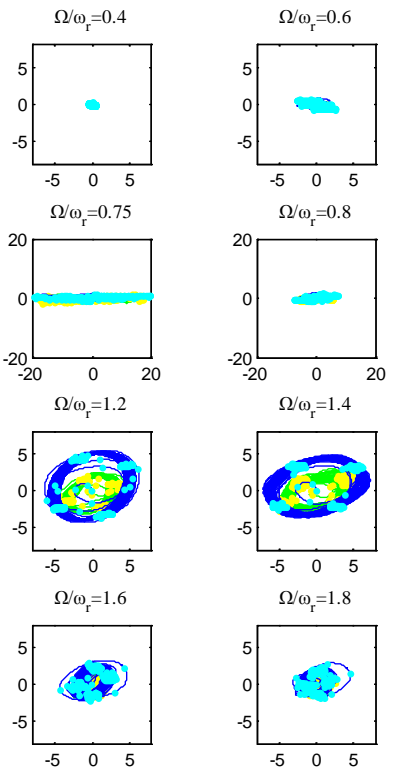

Fig.4. Rotor/stator trajectory, rotor is in blue, stator is in green (wing DOF is considered)
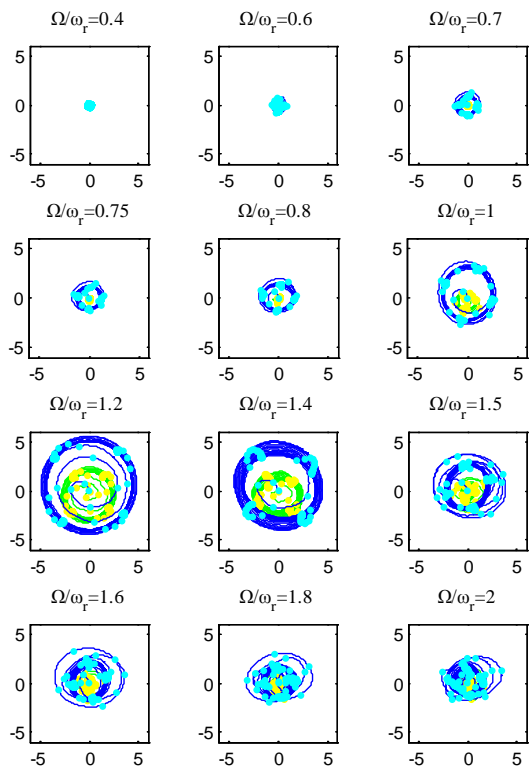

Fig.5. Rotor/stator trajectory (wing DOF is ignored) 
In Fig.4 to Fig.7 of trajectory and spectrum, the unit of all trajectories is [mm], the unit of all frequency in spectrum is $\Omega / \omega_{r}$, and amplitude is in [dB]. Unless special instruction, the broken line indicating spectrum of frequency corresponds to an integer multiple of vibration frequency. A positive frequency indicates forward rotation and a negative frequency means reversed rotation. Rotor data is plotted in blue, and stator's is in green. Considering the need for recording the high-frequency components, there are hundreds of turns in each time course. In order to avoid missing the record points, each time course will include an integer multiple of the vibration cycle number. When the phase of the excitation force passes through $0^{\circ}$, the positions of rotor and stator will be highlighted so that the cycle characteristics can be shown better.

By comparing Fig.4 and Fig.5, it is shown that when wing DOF is considered, as the rotation speed changes from $\Omega / \omega_{r} \approx 0.4$ to $\Omega / \omega_{r} \approx 0.8$, a big jump of rotor/stator trajectory has occurred in one direction, which indicates that vibrations of rotor/stator vibration and wing have coupled at this time, which has aroused a great amplitude. As the rotation speed continues to increase, the variance of rotor/stator trajectory converges to stabilize. Meanwhile, when wing DOF is ignored, the amplitude of rotor/stator trajectory reaches the maximum value around $\Omega / \omega_{r} \approx 1.2$, while the amplitude decreases when rotation speed continues to increase, compared to the case when wing DOF is considered, the amplitude doesn't reach to maximum when rotation speed is in the range from $\Omega / \omega_{r} \approx 0.4$ to $\Omega / \omega_{r} \approx 2$. It is also confirmed by the previous figure (Fig. 3 ) that the existence of wing DOF will arouse vibration of the entire wing/engine system at low frequencies. Thus, the frequency arousing maximum amplitude appears likely to coincide with the frequency of windmilling in the case that wing DOF is considered.
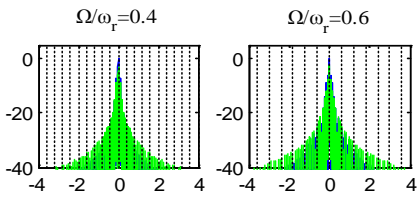

$\Omega / \omega=0.7$
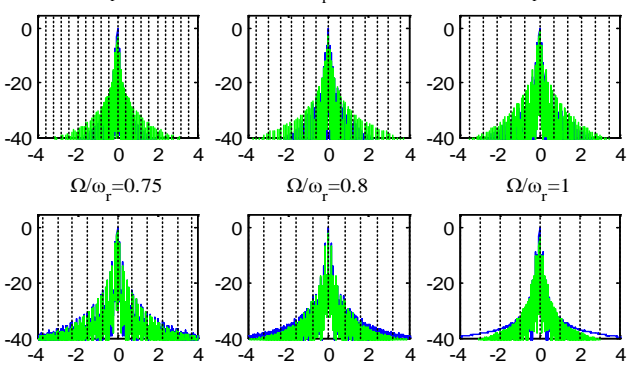

$\Omega / \omega_{\mathrm{r}}=1.2$

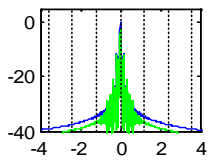

$\Omega / \omega_{\mathrm{r}}=1.4$

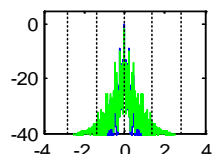

$\Omega / \omega_{\mathrm{r}}=1.8$
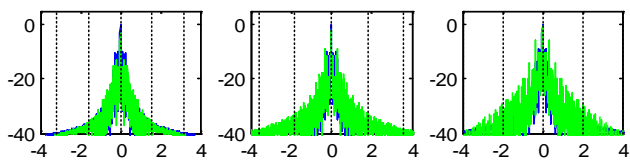

Fig.6. Response of rotor and stator system with consideration of wing DOF

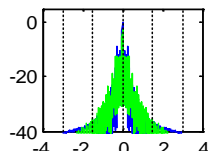

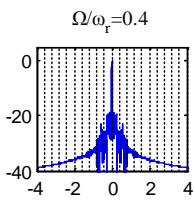

$\Omega / \omega_{\mathrm{r}}=0.6$

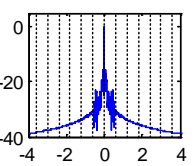

$\Omega / \omega_{\mathrm{r}}=0.8$
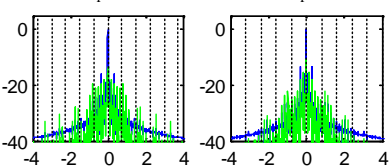

$\Omega / \omega=1.4$
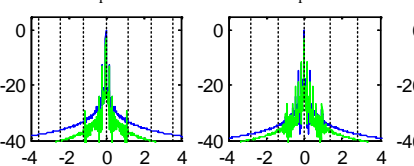

$\Omega / \omega_{\mathrm{r}}=1.8$

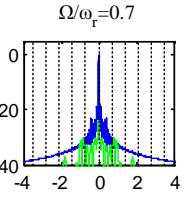

$\Omega / \omega_{\mathrm{r}}=1$

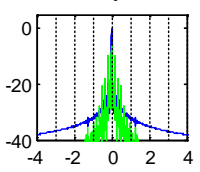

$\Omega / \omega=1.5$

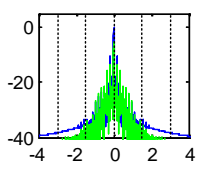

$\Omega / \omega_{\mathrm{r}}=2$

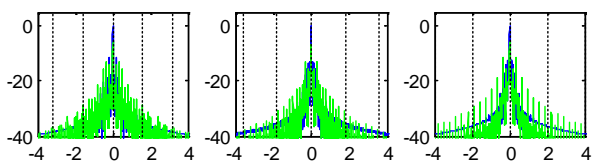

Fig.7. Response of the 4 DOFs rotor and stator system without wing DOF

Comparison of the frequency response in Fig.6, 7 shows that the frequency distribution is very dense when the wing DOF is considered. Due to the influence of wing, compared to the stator amplitude in each case of various frequencies, the presence of wing DOF makes the proportion of stator vibration greater.

In addition, in the two cases that the system contains the wing DOF and not, although the existence of wing will change the rotor and stator vibration, there is no new non-linear behavior.

The other difference of the two cases is that the rotor and stator will remain in contact in a larger interval of rotation speed. Whether the vibration frequency is higher or lower than shaft speed, frequency response will fill the entire spectrum range. The rotor and stator will contact more frequently, which may cause further failure and increase the possibility of serious accidents. 


\section{Conclusion}

By establishing the motion equations of rotor/stator system containing wing DOF and analyzing the rotor/stator trajectory and the frequency response from the simulation result, the following conclusions can be drawn:

1) In the case of asymmetric geometry/stiffness, due to the existence of wing DOF, great vibration amplitude of the wing/engine system was excited early around $\Omega / \omega_{r} \approx 0.75$, which validate further the possibility of catastrophic consequences caused by windmilling imbalance;

2) Compared to the case without wing DOF, although the vibration amplitude of rotor/stator changes, there is no new non-linear behavior, thus during the engine pylons damping design, the impact of wing DOF can be ignored temporarily;

3) Due to the existence of wing, rotor and stator will keep contact in a larger interval of rotation speed, which makes the dangerous speed of aircraft in windmilling expand to a greater range, it will also provide a new reference to the revision of the relevant continuous airworthiness terms of aircraft.

\section{Acknowledgement}

The authors would like to thank Dr. Wang Jianjun for the support of this work through Mistuned Bladed Disk program. This work is supported by the Science Foundation of Civil Aviation University of China (08QD15X), the Science Foundation of Aviation (MHRD20130221) and the Fundamental Research Funds for the Central Universities (ZXH2012J003).

\section{References}

[1] Wang Zhanxue, Wang Yongjie, Qiao Weiyang, Liu Wen. Extrapolating component maps into the low speed and simulation of windminlling performance of turbofan engine [J]. Journal of Propulsion Technology, 2006, 27(2): 146.

[2] Luo Haitao. Dynamic study of single and dual rotor with rub-impact fault [D].Northeastern University, 2009:2-3.

[3] Gotz von Groll. Windmilling in aero-engines[D].Imperial College of Science, Technology \& Medicine University of London, 2000: 6-17,23-25.

[4] An X L, Zhou J Z, Xiang X Q. Dynamic response of a rub-impact rotor system under axial thrust [J]. Archive of Applied Mechanics, 2009, 79(11):1009-1018.

[5] Crandall S H. Nonlinearities in rotor dynamics [J]. Janos Bolyai Mathematical Society, 1987:44-56

[6] Isaksson J L. On the dynamics of a rotor interacting with non-rotating parts [D]. Licentiate's thesis, Linkoping University. 1994: 31-39.

[7] Sun Zhengce, Xu Jiangxue, Gong Pulin. Research of the rub-impact behavior of rotor system [J]. Journal of Vibration Engineering, 2000,13(03):154-160.

[8] Mohamed A A, Mohamed E E, Mohamed G Z. Effect of partial rotor-to-stator rub on shaft vibration [J]. Journal of Mechanical Science and Technology, 2009, 23(1):180-182. 